\title{
Benefit of Targeted Therapies in Metastatic Colon Cancer in the Ivorian Context
}

\author{
Touré Moctar, Kouassi Kouamé K. Yvon, Séka E. Narcisse, Traoré Kady, Kimso Oumou, \\ Niang D. Rokhaya, Odo B. Adde, Oseni Jelili, Adoubi Innocent \\ Department of Oncology, University Hospital of Treichville, Abidjan, Côte d'Ivoire \\ Email: moukitoure@yahoo.fr
}

How to cite this paper: Moctar, T., Yvon, K.K.K., Narcisse, S.E., Kady, T., Oumou, K., Rokhaya, N.D., Adde, O.B., Jelili, O. and Innocent, A. (2017) Benefit of Targeted Therapies in Metastatic Colon Cancer in the Ivorian Context. Journal of Cancer Therapy, 8, 785-792.

https://doi.org/10.4236/jct.2017.88068

Received: July 14, 2017

Accepted: August 12, 2017

Published: August 15, 2017

Copyright (c) 2017 by authors and Scientific Research Publishing Inc. This work is licensed under the Creative Commons Attribution International License (CC BY 4.0).

http://creativecommons.org/licenses/by/4.0/

\begin{abstract}
In our daily practice, the use of targeted therapies (Bevacizumab and rarely Cetuximab) in metastatic colic cancers is recent. Very few patients benefit from these therapies because of their high cost. In a cohort of 68 patients who received these therapies, a retrospective and prospective study was conducted over three years period (1 January 2013 to 31 December 2015) to evaluate their benefit in terms of quality of life, tolerance and overall survival. These targeted therapies provided a significant clinical and biological gain with acceptable toxicities (most often resolving spontaneously). Moreover, they have allowed a significant improvement in overall survival in first line metastatic treatment. The limiting factor remains their extremely high cost and therefore inaccessibility to the majority of our patients.
\end{abstract}

\section{Keywords}

Cancer-Colon-Metastasis-Targeted Therapy

\section{Introduction}

The increasingly accurate knowledge of colon oncogenesis has enabled the development of targeted therapies [1] [2]. These are generally anti-angiogenic and anti-EGFR antibodies which, in western series, have shown certain activity in first line metastatic colon cancers [3] [4]. These molecules, for the most part, are inaccessible for most patients in sub-Saharan Africa in general and in Côte d'Ivoire in particular. Very few scientific studies have evaluated the benefits of these targeted therapies (TTs) in our current practice. The objective of this study was to contribute to the improvement in management of metastatic colon cancer in the Ivorian context. Specifically, the aim was to identify the contribution of these TTs in terms of response, tolerance and overall survival. 


\section{Patients and Methods}

This was a preliminary retrospective and prospective study over a three years period (1 January 2013 to 31 December 2015). This study oversaw 68 cases (mean age 49 years) of histologically confirmed metastatic colon cancer in the Oncology Department at the University Hospital of Treichville in Abidjan. The recruitment of patients took place not only at Treichville university hospital, but at the two other University Hospital Centers in Abidjan, namely Cocody and Yopougon. Were included into our study, patients who had at least six courses of chemotherapy including some targeted therapy. Also, patients whose cures were done every 14 days. Criteria for non-inclusion included patients with incomplete records and those with a WHO performance status score of 3 and over.

The data collection was recorded on a question sheet containing the socioeconomic, demographic and other parameters required for our study. For the retrospective aspect, data was collected from patient files seen respectively in hospitalization and in consultation. The operative reports and the histological results recorded in the registries of the pathology departments of Abidjan's University Hospitals were also taken into account. As for the prospective aspect, a thorough interrogation allowed to assess the socio-demographic characteristics, the weight loss percentage calculated from the weight ratio at the time of diagnosis over the initial weight. It also heplped to evaluate functional signs. A rigorous physical examination and endoscopic explorations (total colonoscopy) made it possible to suspect the colonic malignancy. It was during this period that a biopsy with anatomo-pathological examination was systematically performed. The diagnosis was made on the histology of the surgical specimen. Confidentiality was respected throughout the methodological approach. Following the confirmation of a colon adenocarcinoma, a complete workup including morphological explorations (thoraco-abdomino-pelvic computed tomography) allowed staging by the Union for International Cancer Control (UICC) TNM (Tumor Nodes Metastasis) system [5].

Concerning our Treatment Methodology, a pre-therapeutic assessment was systematically undertaken with the aim to minimizing possible toxicities. These included complete blood count, uremia, serum creatinine and transaminasemia. As for tumor markers (ACE and CA 19-9), they were done for further monitoring. All patients received a palliative chemotherapy protocol, including some targeted therapy. These protocols were Folfox4-Bevacizumab, Folfox4-Cetuximab, Folfox 4 or Folfiri, Fufol-Plattine. The non-mutated K-RAS status was a prerequisite for the administration of Cetuximab.

Response was evaluated according to the RECIST 2000 criteria defining complete response (CR), partial response (PR), tumor stabilization (TS) and tumor progression (TP) [6]. Tolerance was evaluated according to the World Health Organization (WHO) criteria for toxicity of anticancer drugs. Survival was evaluated using the Kaplan Meier method [7]. 


\section{Results}

68 patients met the criteria for inclusion with an average age of 49 years [29 75]. There was a male predominance coming mostly from urban areas. Patients who benefited from these different protocols were most likely to have an average to high socio-economic level and their main source of funding came from private insurance (Table 1). About $86.6 \%$ of our patients consulted after 6 months of beginning of symptoms. Pain was the most common clinical symptom encountered in about $80.9 \%$ of cases. Left colon was the most frequent primary tumor site $(63.2 \%$ of cases). Metastatic localization was most often hepatic (70.6\% of cases). As for the histological type, it was in $72 \%$ of cases a Lieberkhünien adenocarcinoma (Table 2). All protocols combined showed a significant clinical as well as biological gain in post-treatment (Table 3, Figure 1).

Table 1. Socio-demographic characteristic.

\begin{tabular}{|c|c|c|c|}
\hline \multicolumn{2}{|c|}{ Socio-demographic characteristic } & \multirow{2}{*}{$\frac{\text { Number (n) }}{9}$} & \multirow{2}{*}{$\begin{array}{c}\text { Percentage (\%) } \\
13.2\end{array}$} \\
\hline \multirow{5}{*}{ Age (years) } & $30-39$ & & \\
\hline & $40-49$ & 14 & 20.5 \\
\hline & $50-59$ & 21 & 30.9 \\
\hline & $60-69$ & 13 & 19.2 \\
\hline & $\geq 70$ & 11 & 16.2 \\
\hline \multirow{2}{*}{ Gender } & Male & 48 & 70,6 \\
\hline & Female & 20 & 29,4 \\
\hline \multirow{2}{*}{ Residency } & Urban & 35 & 51,5 \\
\hline & Rural & 33 & 48,5 \\
\hline \multirow{3}{*}{ Socio-econ level } & Low & 7 & 10,3 \\
\hline & Average & 45 & 66,2 \\
\hline & High & 16 & 23,5 \\
\hline \multirow{3}{*}{ Financial provider } & Insurance & 51 & 75 \\
\hline & Relative & 7 & 10 \\
\hline & Patient & 10 & 15 \\
\hline
\end{tabular}

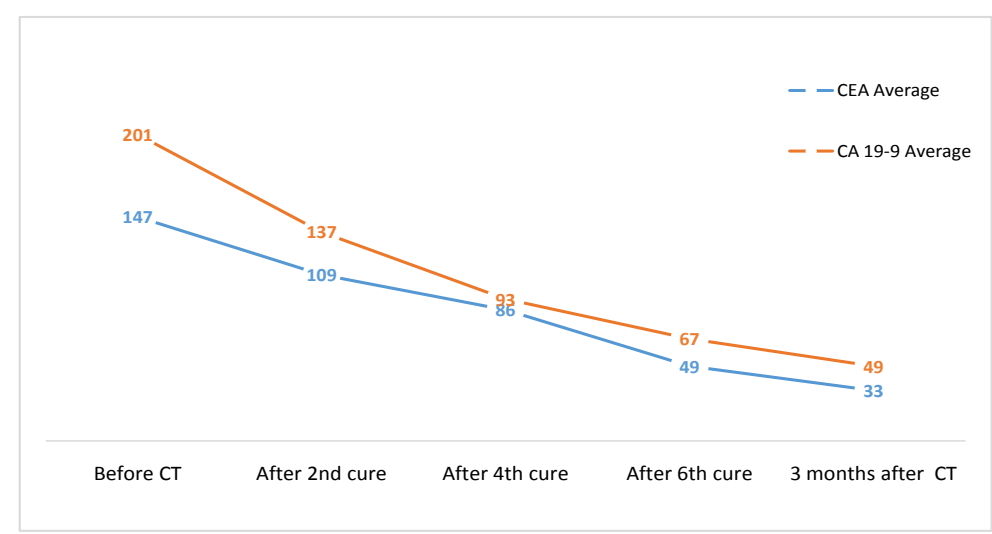

Figure 1. Evolution of ACE and CA 19 - 9 average rates during and after chemotherapy. 
Table 2. Clinico-pathologic characteristics.

\begin{tabular}{|c|c|c|}
\hline Clinico-pathologic characteristics & Number $(n)=68$ & $\%$ \\
\hline \multicolumn{3}{|c|}{ Delay until consultation } \\
\hline$<6$ months & 9 & 13.2 \\
\hline$[6]-[12]$ & 14 & 20.6 \\
\hline$>12$ months & 45 & 66.2 \\
\hline \multicolumn{3}{|c|}{ Motif $f$ consultation } \\
\hline Abdominal pain & 55 & 80.9 \\
\hline Motility disorder & 14 & 20.6 \\
\hline Hemorrhage & 9 & 13.2 \\
\hline Occlusion & 23 & 33.8 \\
\hline Perforation & 11 & 16.2 \\
\hline \multicolumn{3}{|c|}{ WHO performance status } \\
\hline 1 & 18 & 26.5 \\
\hline 2 & 50 & 73.5 \\
\hline \multicolumn{3}{|c|}{ Tumor location } \\
\hline Right colon & 25 & 36.8 \\
\hline Left colon & 43 & 63.2 \\
\hline \multicolumn{3}{|c|}{ Method of diagnostic } \\
\hline Colonoscopy + Biopsy & 33 & 48.5 \\
\hline Surgical resection & 35 & 51.5 \\
\hline \multicolumn{3}{|c|}{ Metastatic sites } \\
\hline Liver & 48 & 70.6 \\
\hline Lungs & 11 & 16.2 \\
\hline Other & 9 & 13.2 \\
\hline \multicolumn{3}{|c|}{ Histological types } \\
\hline Lieberkhünian carcinoma & 49 & 72 \\
\hline colloid mucinous carcinoma & 13 & 19.1 \\
\hline Signet ring cell carcinoma & 6 & 8.9 \\
\hline
\end{tabular}

Protocols including targeted therapies provided the most significant therapeutic responses compared to chemotherapy protocols alone (Table 4). Tumor progression was most frequently observed with the Fufol-platine protocol. No complete response was observed in our study. Hematological toxicities, of which, anemia (51.5\% of cases) was the most frequently encountered. Leukoneutropenia occurred in approximately $45.6 \%$ of cases. Neutropenia was mostly grade I (45\%) and II (39\%). Grade III neutropenia occurred in 16\% of cases. Digestive toxicities were mainly represented by diarrhea ( $29.5 \%$ of cases). Other toxicities included mucositis $(33.9 \%)$, skin rash (5.9\% of cases mainly for targeted therapies) and peripheral neuropathies (36.8\% mainly for Oxaliplatin) (Table 5). 
Table 3. Clinical response after treatment.

\begin{tabular}{cccccc}
\hline \multirow{2}{*}{ Clinical Characteristics } & \multicolumn{2}{c}{ Before Chemo } & \multicolumn{2}{c}{ After 6 cures of chemo } & \multirow{2}{*}{ p } \\
\cline { 2 - 4 } & Number (n) & $\%$ & Number (n) & $\%$ & \\
\hline Who performance status & 21 & 31 & 52 & 76.5 & $<0.01$ \\
1 & 47 & 69 & 16 & 23.5 & $<0.01$ \\
Symptoms & & & & & \\
Abdominal pain & 56 & 82 & 12 & 17.6 & $<0.01$ \\
Motility disorders & 15 & 22 & 3 & 4.4 & $<0.01$ \\
Hemorrhage & 9 & 13 & 2 & 3 & $<0.01$ \\
Weight loss & 31 & 46 & 11 & 16 & $<0.01$ \\
\hline
\end{tabular}

Chemo: Chemotherapy, WHO: World Health Organization.

Table 4. Clinical response according to the different protocols.

\begin{tabular}{cccccc}
\hline Protocoles & CR $\%$ & PR $\%$ & TS $\%$ & TP \% & Number (n) \\
\hline Folfox-Bev & 0 & $37 \%$ & $33 \%$ & $30 \%$ & 19 \\
Folfox-Cetux & 0 & $27 \%$ & $38 \%$ & $35 \%$ & 5 \\
Folfox/Folfiri & 0 & $13 \%$ & $30 \%$ & $67 \%$ & 20 \\
Fufol-Platine & 0 & $8 \%$ & $17 \%$ & $75 \%$ & 24 \\
\hline
\end{tabular}

CR: Complete response, PR: Partial response, TS: Tumor stabilization, TP: Tumor progression, Bev: Bevacizumab, Cetux: Cetuximab.

Survival was better in patients with protocols that included targeted therapies compared to chemotherapy alone. Patients were followed over an 18 months period. The survival was $63 \%$ and $62 \%$ respectively for Folfox4-Bevacizumab and Folfox4-Cetuximab. The Folfox4 protocol in terms of survival gave better survival compared to FUFOL-Platine ( $40 \%$ versus $20 \%$ at 18 months). There was no significant difference in Folfox4-Bevacizumab versus Folfox 4 Cetuximab (Figure 2 and Table 6).

\section{Discussion}

Bevacizumab followed by Cetuximab are the most widely used targeted therapies in our daily practice. These molecules have certain activity in first line metastatic colon cancers [8] [9]. Combined with chemotherapy, they have improved the quality of life and comfort of our patients. Indeed, prior to the advent of targeted therapies, the combination of oxaliplatin (Folfox4) or irinotecan (Folfiri) with fluorouracil had already influenced the therapeutic approach of metastatic colon cancer by providing options for patients resistant to more conventional treatments. The introduction of Bevacizumab (antiVEGF) resulted in rapid vascular regression and Cetuximab (anti-EGFR in non-mutated K-ras metastatic colon cancer) potentiating the cytotoxic effect of chemotherapy [10] [11]. Other monoclonal antibodies have also been the subject of therapeutic trials in metastatic 
Table 5. Toxicity associated to different protocols.

\begin{tabular}{cccccc}
\hline \multirow{2}{*}{ Toxicity } & \multicolumn{2}{c}{ Folfox-Bev } & Folfox-Cetux Folfox ou Folfiri Fufol-Platine & \multirow{2}{*}{ Total } \\
\cline { 2 - 4 }$(\mathrm{n}=19)$ & $(\mathrm{n}=5)$ & $(\mathrm{n}=20)$ & $(\mathrm{n}=24)$ & \\
\hline $\begin{array}{c}\text { Hematologic toxicities } \\
\text { Anemia }\end{array}$ & 7 & 2 & 14 & 12 & $35(51.5 \%)$ \\
Thrombopenia & 0 & 1 & 4 & 0 & $5(7.4 \%)$ \\
Leukopenia & 10 & 3 & 11 & 7 & $31(45.6 \%)$ \\
Digestive toxicities & & & & & \\
Diarrhea & 2 & 3 & 9 & 6 & $20(29.5 \%)$ \\
Vomiting & 2 & 1 & 5 & 4 & $12(17.6 \%)$ \\
Other toxicities & & & & & \\
Mucositis & 6 & 2 & 7 & 8 & $23(33.9 \%)$ \\
Cutaneous & 0 & 4 & 0 & 0 & $4(5.9 \%)$ \\
Peripheral neuropathies & 13 & 2 & 9 & 1 & $25(36.8 \%)$ \\
\hline
\end{tabular}

Table 6. Evolution of chemo-induced neutropenia.

\begin{tabular}{ccccl}
\hline Neutropenia & Number (n) & Percentage (\%) & Treatment & Outcome \\
\hline Grade I & 14 & 45 & monitoring & Favorable \\
Grade II & 12 & 39 & VIT, Corticotherapy & Favorable \\
Grade III & 5 & 16 & GF, BI ATB, VIT & Favorable \\
Total & 31 & 100 & & \\
\hline
\end{tabular}

FC: growth factor; BI ATB: bi-antibiotic therapy; VIT: vitamin therapy.

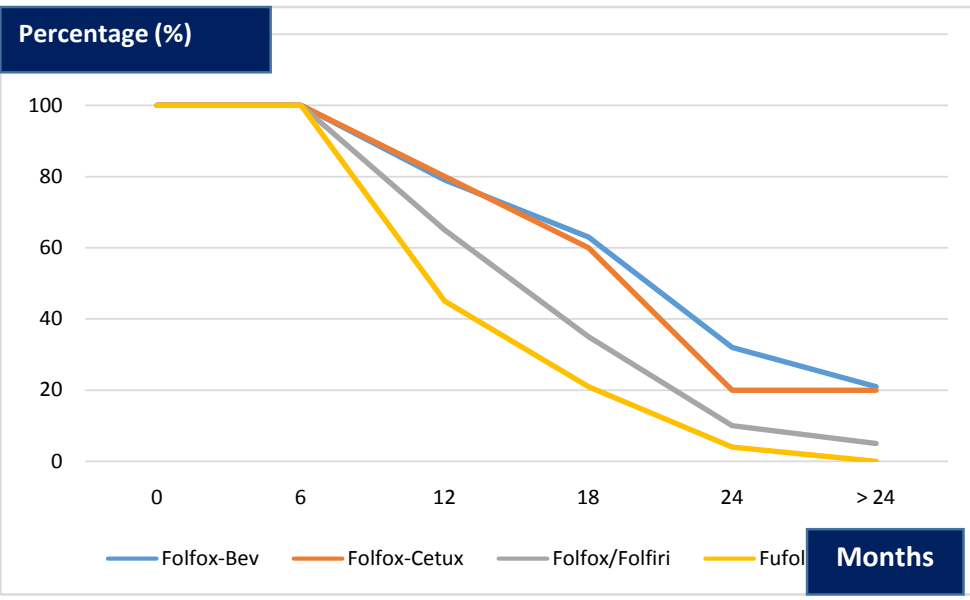

Figure 2. Overall survival according to different protocols.

colon cancer. This is Panitumumab (not used in our series) an anti-EGFR which leads to tumor responses along the lines of $13 \%$ to $20 \%$ in patients with recurrent or refractory colon cancer [12].

The observed toxicities were generally low grade and therefore most often resolved spontaneously. For those patients with febrile leukoneutropenia, man- 
agement was based on standard options and recommendations. This standard included antibiotic therapy (Fluoroquinolone and cephalosporin) associated with hematopoietic growth factors (Filgrastine or lenograstim) [13] [14]. The classic high blood pressure associated with bevacizumab and described in the literature had not been observed in our series. However, it is always necessary to control the blood pressure and test for possible proteinuria during the treatment. As for cetuximab, the most disabling toxicity was cutaneous, with acneiform eruption sometimes requiring treatment with systemic tetracyclines [15]. This was observed in almost all of our patients under Cetuximab.

The results of our study in terms of overall survival are comparable to the works of Hurwitz et al., who found an overall survival of 16 months [16]. The improvement of survival rate is generally due to the combination of chemotherapy and targeted therapies. Bevacizumab has been shown to be effective in combination with FOLFIRI (irinotecan +5 FU bolus + folinic acid) compared to patients treated with chemotherapy alone.

\section{Conclusion}

Therapeutic protocols (targeted therapy and/or chemotherapy) were instituted in 68 patients with metastatic colon cancer. Combined with chemotherapy, targeted therapies have improved the quality of life and comfort of our patients. According to the RECIST criteria 2000, the most significant objective responses were observed with these different associations. However, these treatments can induce toxicities which generally resolved spontaneously or with treatment. Moreover, they have allowed first line metastatic colon cancer treatment to improve overall survival. The limiting factor remains their extremely high cost and therefore not readily accessible to patients. This study is a plea to public authorities to facilitate access to these innovative therapies that have revolutionized the prognosis of metastatic colon cancers.

\section{Conflict of Interest}

None.

\section{References}

[1] Vilar, E., et al. (2010) Les 2 principales voies de carcinogenèse colorectale: Voie CIN (ou LOH); voie MSI. Nature Reviews Clinical Oncology, 7, 153-162. https://doi.org/10.1038/nrclinonc.2009.237

[2] Laurent-Puig, P., Agostini, J., Maley, K., et al. (2010) Oncogenèse colorectale. Bulletin du Cancer, 97, 1311-1321.

[3] Patel, G.S. and Karapetis, C.S. (2013) Personalized Treatment for Advanced Colorectal Cancer: KRAS and Beyond. Cancer Management and Research, 5, 387-400.

[4] Douillard, J.Y., Oliner, K.S., Siena, S., et al. (2013) Panitumumab-Folfox4 Treatment and RAS Mutations in Colorectal Cancer. The New England Journal of Medicine, 369, 1023-1034. https://doi.org/10.1056/NEJMoa1305275

[5] Edwin, R.F., Richard, S., Alka, P., et al. (1989) Dukes' Classification Revisited. Findings from the National Surgical Adjuvant Breast and Bowel Projects (Protocol 
r-01). Cancer, 64, 2354-2360.

https://doi.org/10.1002/1097-0142(19891201)64:11<2354::AID-CNCR2820641127> 3.0.CO;2-\#

[6] Duffaud, F. and Therasse, P. (2000) Nouvelles Recommandations Pour l'évaluation de la réponse tumorale dans les tumeurs solides. Bulletin du Cancer, 87, 881-886.

[7] Kaplan, E.L. and Meier, P. (1958) Non Parametric Estimation from Incomplete Observations. Journal of the American Statistical Association, 53, 457-481. https://doi.org/10.1080/01621459.1958.10501452

[8] Meyerhardt, J.A. and Mayer, R.J. (2005) Systemic Therapy for Colorectal Cancer. The New England Journal of Medicine, 352, 476-487. https://doi.org/10.1056/NEJMra040958

[9] Tol, J., Koopman, M., Cats, A., et al. (2009) Chemotherapy, Bevacizumab, and Cetuximab in Metastatic Colorectal Cancer. The New England Journal of Medicine, 360, 563-572. https://doi.org/10.1056/NEJMoa0808268

[10] De Roock, W., Claes, B., Bernasconi, D., et al. (2010) Effects of KRAS, BRAF, NRAS, and PI3KA Mutations on the Efficacy of Cetuximab plus Chemotherapy in Chemotherapy-Refractory Metastatic Colorectal Cancer: A Retrospective Consortium Analysis. The Lancet Oncology, 11, 753-762.

[11] Lenz, H.J., Van Cutsem, E., Khambata-Ford, S., et al. (2006) Multicenter Phase II and Translational Study of Cetuximab in Metastatic Colorectal Carcinoma Refractory to Irinotecan, Oxaliplatin, and Fluoropyrimidines. Journal of Clinical Oncology, 24, 4914-4921. https://doi.org/10.1200/JCO.2006.06.7595

[12] Hecht, J.R., Patnaik, A., Berlin, J., et al. (2007) Panitumumab Monotherapy in Patients with Previously Treated Metastatic Colorectal Cancer. Cancer, 110, 980-988. https://doi.org/10.1002/cncr.22915

[13] Bouché, O., Scaglia, E., Reguiai, Z., Singha, V., Brixi-Benmansour, H. and Lagarde, S. (2009) Biothérapies ciblées en cancérologie digestive: Prise en charge de leurs effets secondaires. Gastroenterologie Clinique et Biologique, 33, 306-322.

[14] Tejpar, S., Peeters, M., Humblet, Y., et al. (2007) Phase I/II Study of Cetuximab Dose-Escalation in Patients with Metastatic Colorectal Cancer (mCRC) with no or Slight Skin Reactions on Cetuximab Standard Dose Treatment (EVEREST): Pharmacokinetic (PK), Pharmacodynamic (PD) and Efficacy Data. ASCO Meeting Abstracts, 25, 4037.

[15] Scartozzi, M., Bearzi, I., Berardi, R., et al. (2004) Epidermal Growth Factor Receptor (EGFR) Status in Primary Colorectal Tumors Does Not Correlate with EGFR Expression in Related Metastatic Sites: Implications for Treatment with EGFRTargeted Monoclonal Antibodies. Journal of Clinical Oncology, 22, 4720-4726. https://doi.org/10.1200/JCO.2004.00.117

[16] Hurwitz, H., Fehrenbacher, L., Novotny, W., et al. (2004) Bevacizumab plus Irinotecan, Fluorouracil, and Leucovorin for Metastatic Colorectal Cancer. The New England Journal of Medicine, 350, 2335-2342. https://doi.org/10.1056/NEJMoa032691 
Submit or recommend next manuscript to SCIRP and we will provide best service for you:

Accepting pre-submission inquiries through Email, Facebook, LinkedIn, Twitter, etc. A wide selection of journals (inclusive of 9 subjects, more than 200 journals)

Providing 24-hour high-quality service

User-friendly online submission system

Fair and swift peer-review system

Efficient typesetting and proofreading procedure

Display of the result of downloads and visits, as well as the number of cited articles Maximum dissemination of your research work

Submit your manuscript at: http://papersubmission.scirp.org/

Or contact jet@scirp.org 